Revue des patrimoines

15 | 2011

Le patrimoine des guides : lectures de l'espace urbain européen

\title{
Les formes du voyage : cartes et espaces des guides de voyage
}

Nicolas Verdier

\section{(2) OpenEdition}

Journals

Édition électronique

URL : http://journals.openedition.org/insitu/573

DOI : 10.4000/insitu.573

ISSN : 1630-7305

Éditeur

Ministère de la culture

\section{Référence électronique}

Nicolas Verdier, «Les formes du voyage : cartes et espaces des guides de voyage », In Situ [En ligne], 15 | 2011, mis en ligne le 29 juin 2011, consulté le 20 avril 2019. URL : http://journals.openedition.org/ insitu/573 ; DOI : 10.4000/insitu.573

Ce document a été généré automatiquement le 20 avril 2019

\section{(c) $($ () $\ominus$}

In Situ Revues des patrimoines est mis à disposition selon les termes de la licence Creative Commons Attribution - Pas d'Utilisation Commerciale - Pas de Modification 4.0 International. 


\title{
Les formes du voyage : cartes et espaces des guides de voyage
}

\author{
Nicolas Verdier
}

1 Sans remonter ${ }^{1}$ jusqu'à la table de Peutinger, on peut dire qu'en France, depuis qu'en 1645 J. Boisseau a fabriqué une carte reproduisant les itinéraires de la Guide des chemins de France de Charles Estienne (1552) la relation entre guide de voyage et carte ne semble pas s'être démentie. Ce sont les modalités de la relation qui se sont en revanche diversifiées, faisant dans le même temps évoluer, voire varier, l'appréhension de ce que Jules Gritti appelait en 1967 «l'espace touristique » - en décryptant les hiérarchies entre lieux et itinéraires plus ou moins importants ${ }^{3}$. De façon à revenir sur cette question de l'espace des guides, je souhaiterais tout d'abord me concentrer sur la nature des cartes contenues dans quelques guides de l'époque moderne et de l'époque contemporaine, avant de tenter d'analyser en quoi les différentes modalités du cartographique sont en relation avec la structure même de ces ouvrages voués aux voyageurs.

Avant toute chose, il convient de définir quelques éléments du corpus. L'expression même de "guide de tourisme » est extrêmement lourde de sens : l'idée de «guide» renvoie aussi bien à un être humain, souvent originaire de la région qui conduit les voyageurs vers tel ou tel lieu, qu'à un livre, qui indique l'ensemble des points de passage possibles pour lier points de départ et d'arrivée $e^{4}$. Aux Guides-Cicérone, je préfèrerais ici les livres dont les aspects éminemment pratiques ont très tôt été augmentés des éléments permettant d'étendre le bon repérage des voyageurs dans les mondes économiques (présence d'industrie, d'artisanat, de banques...) et/ou culturels (présence de monuments, localisation d'événements historiques, collections d'œuvres d'art, aspects pittoresques...), voire plus prosaïquement matériels (hôtelleries, restaurants, lieux de louage de véhicules... horaires et conditions des transports en commun). Comparer un Livre de Poste de 1708 à un Guide Murray de 1867 montre l'ampleur des changements. Le nombre de pages suffit d'ailleurs puisque pour des formats relativement proches - encore que les « livres de poste » soit des in-16, alors que le suivant est un in- $8^{\circ}-$ le Livre de Poste de 1708 compte 43 pages, le Murray pour la France de 1867 en compte 685. Le premier, très 
fonctionnel, ne concerne que les aspects matériels du déplacement par la poste aux chevaux. Le second, beaucoup plus détaillé couvre tout un spectre de savoirs et d'usages que le voyageur peut être amené à utiliser. Quant à l'idée de tourisme, elle est en partie rattachée selon l'époque à l'idée de "Grand Tour ", ou à celle de «tourisme de masse " qui sont des pratiques peu commensurables. Elle offre cependant un large choix que je limiterai ici aux publications vers un large public.

\section{Cartographies des voyages}

Tenter une recension complète des cartes présentes dans les différents guides de voyage n'est pas l'objet de cette étude. Posons cependant l'un des éléments de cette présence. Les cartes des guides n'ont pas nécessairement été dressées pour eux. Comme pour les gravures qui passent d'un ouvrage à un autre en fonction des collections de plaques de cuivre des éditeurs, les cartes sont utilisées et réutilisées sans toujours que l'on connaisse les orientations des publications qui ont amené à les produire. Dans bien des cas, elles ne font donc pas corps avec l'ouvrage auquel elles n'appartiennent qu'artificiellement ${ }^{5}$. Dès lors, toute analyse des relations entre cartes et livres apparaît fragilisée. Le choix des seules cartes produites pour des livres particuliers semble par ailleurs illusoire. Comment être certain qu'une carte n'existait pas avant? Mais surtout, la diffusion de modèles de présentation, et donc la nature même de la mise en place de modèles de relation entre cartes et textes passe aussi par la répétition et les emprunts. Reste à utiliser une autre méthode qui est l'étude de la mise en relation entre textes et cartes. Nous commencerons ici par comparer la place de la carte dans une série d'ouvrages :

- le Nouveau voyage de la France de Piganiol de la Force, dans son édition de 1724 ;

- L'indicateur fidèle de Michel et Desnos dans son édition de 1765;

- l'Itinéraire Complet de la France... de Louette édité en 1788 ;

- le Livre de Poste, dans trois éditions successives (1708, 1716 et 1774);

- le Petit itinéraire du chemin de fer de Paris au Havre, paru vers 1853 ;

- le Handbook for travellers in France chez Murray dans son édition de 1867 ;

- le Guide Diamant sur La France d'Adolphe Joanne chez Hachette dans son édition de 1873 ;

- le Guide Bleu pour La France dirigé par Francis Ambrière chez Hachette en 1956.

En dehors de l'Itinéraire complet de la France... de 1788 et du Petit itinéraire de 1853 (qui en fait rassemble trois textes plus anciens, 1845, 1847 et 1853, mais ne semble pas avoir eu de réédition ${ }^{6}$, l'ensemble de ces ouvrages a connu plusieurs éditions successives, parfois concentrées lors d'une aventure éditoriale (Michel et Desnos entre 1761 et 1780), parfois éloignées les unes des autres (Nouveau voyage de la France en 1724, 1740, 1755 et 1780), parfois très régulières (Livres de Poste tous les ans). En outre, tous ces ouvrages sont liés plus ou moins intimement à des moyens de transport depuis les malles-postes jusqu'à l'automobile en passant par les trains. Enfin, chacun de ces ouvrages compte au moins une carte, et parfois plusieurs. 


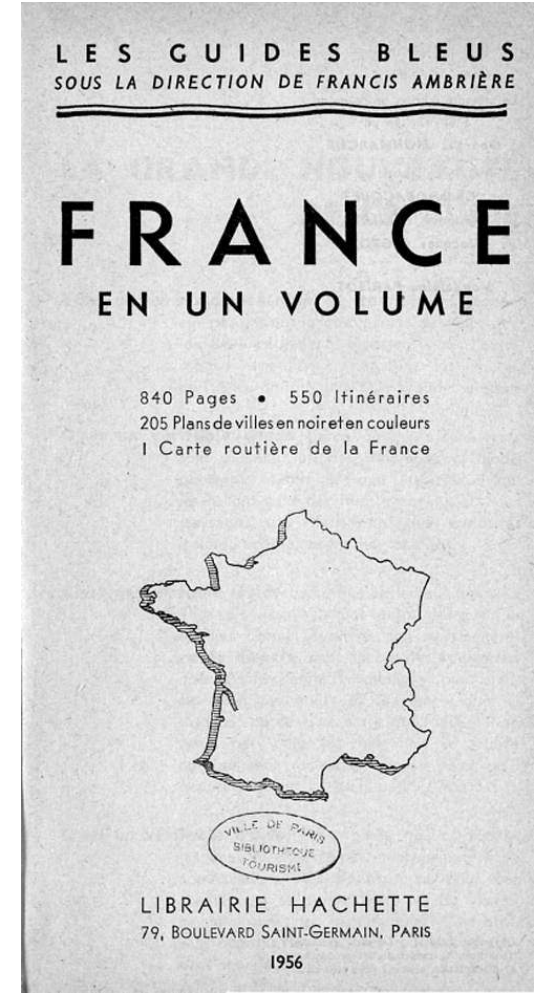

Première page du Guide Bleu pour la France en 1956. France en un volume, Les Guides Bleus. Paris : Hachette, 1956, p. 1

(c) Guide Bleu/France/, Hachette, 1956

5 Il est possible de répartir les articulations entre textes et cartes en trois grands types. Ceux-ci recoupent approximativement des classifications en fonction des formats, ou de la position de la (ou des) carte(s) dans l'ouvrage. Le premier, peut-être le plus complexe à délimiter est la carte exclusivement comme signe. La carte ne sert pas au voyage, elle l'englobe, voire elle prétend donner à voir la portée de l'ouvrage. La page de titre du Guide bleu de la France en est la représentation idéale (fig. $\left.\mathbf{n}^{\circ} \mathbf{1}\right)$. Pour reprendre les mots de Louis Marin, la carte est ici « le simulacre qui permet de s'approprier dans un seul regard la totalité... ${ }^{7}$ » Il ne s'agit pas ici de permettre le repérage, mais plus fortement de figurer le contenu du livre, voire d'ancrer le lecteur dans une posture de voyage. Cela permet aussi peut-être d'en rattacher le contenu à la Géographie. Bien souvent, les auteurs de guide, du moins aux XVIII ${ }^{\mathrm{e}}$ et XIX ${ }^{\mathrm{e}}$ siècles, se disent "Géographes " sans que jamais ensuite le mot ne réapparaisse dans le texte. Les problèmes de délimitation de cette catégorie viennent des limites entre carte proposant une figuration, et carte proposée au lecteur pour se repérer. Que penser en effet d'une carte comme celle introduite dans la Liste générale des Postes de 1716, sachant que sa petite taille (environ $10 \mathrm{~cm}$ de côté) (fig. $\mathbf{n}^{\circ}$ 2), et l'absence du réseau dans son entier ne permettent pas au voyageur d'y trouver des éléments pour se repérer ? À choisir c'est plus la mention, dans un petit cartouche de l'Académie des Sciences - au nord-est de la carte - qui sert ici à placer cet objet du côté des savoirs savants. 
Figure 2

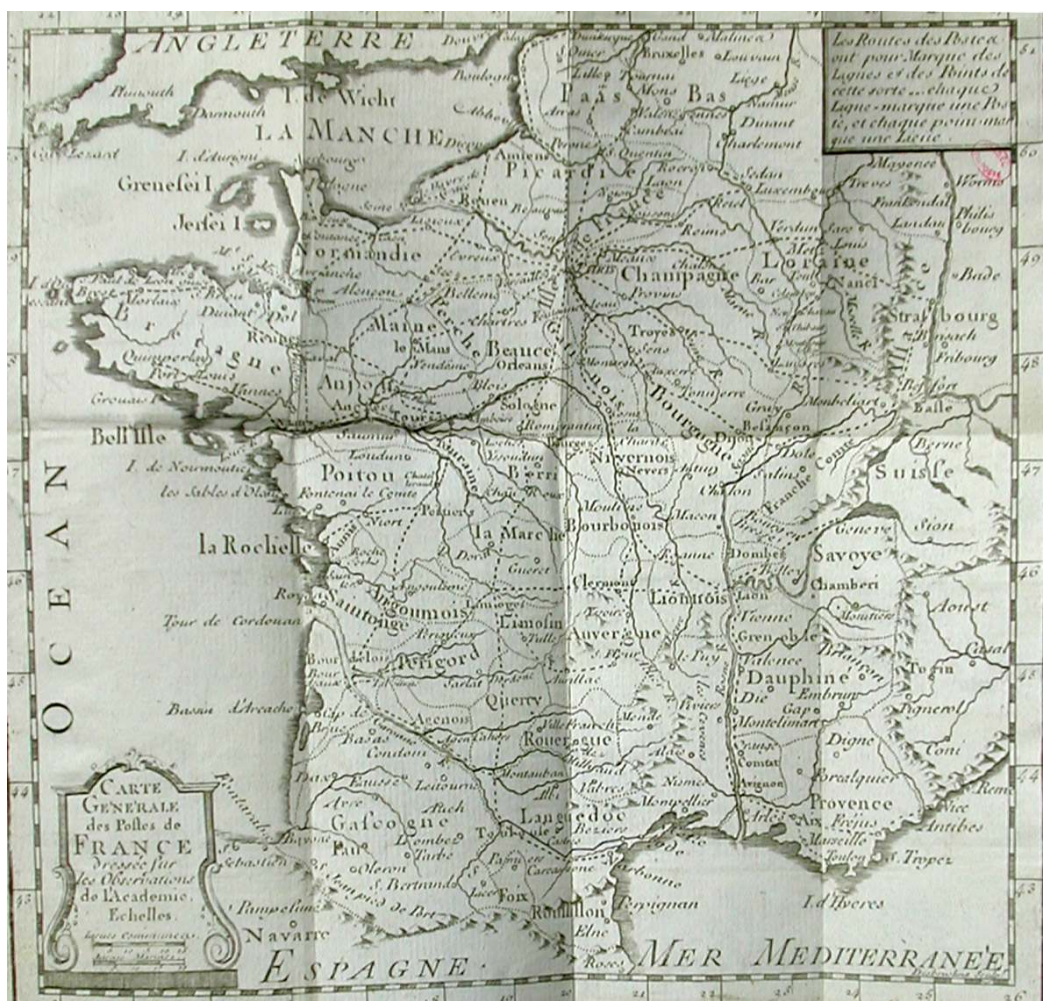

Carte générale des Postes de France, in Liste générale des Postes de France... Paris : Chez le sieur Jaillot, Géographe ordinaire du Roy, 1716.

De même, la carte de Louette, publiée en 1788, si elle offre une remarquable figuration du réseau français - très probablement recopiée sur les cartes des livres de Poste - auquel elle ajoute des routes dans les pays circonvoisins n'est fournie qu'à la fin du second tome de l'Itinéraire complet... (fig. $\mathbf{n}^{\circ} \mathbf{3}$ ). 
Figure 3

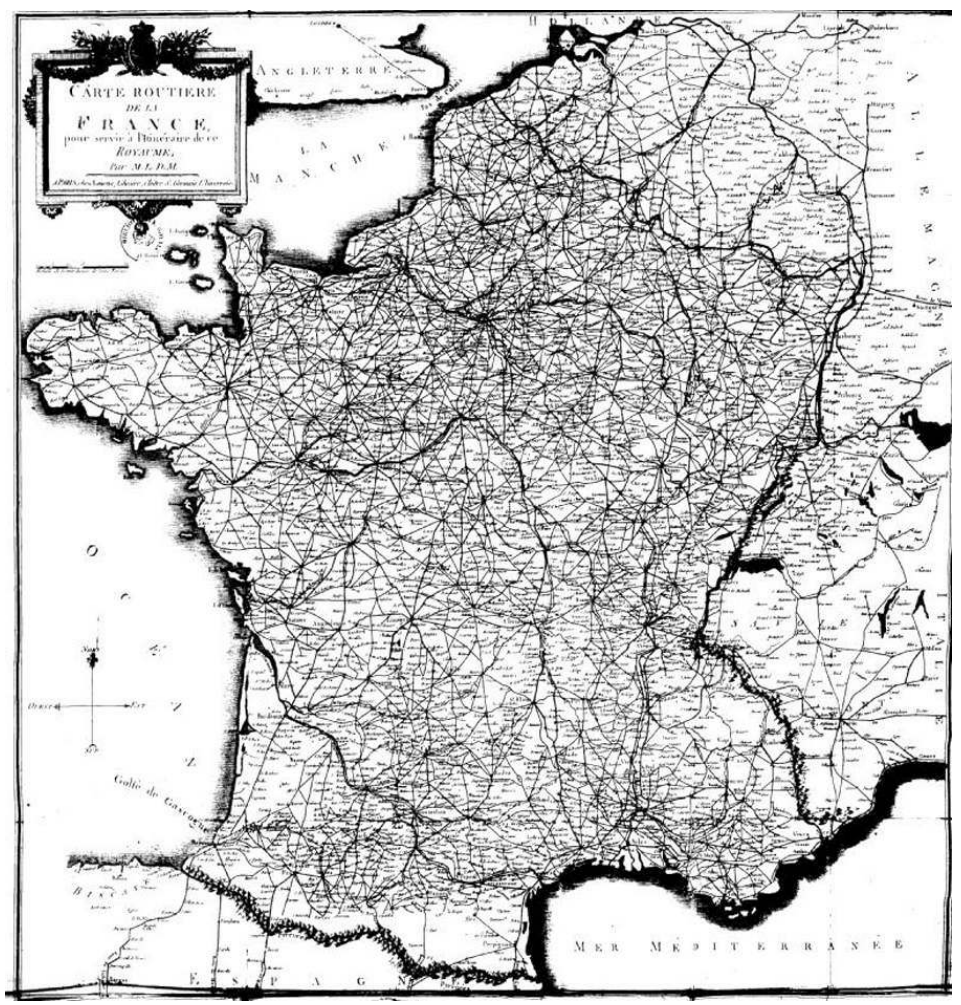

Carte routière de la France, in Itinéraire complet de la France ou tableau général de toutes les routes et chemins de traverse de ce royaume... Orné d'une carte géographique, par MLDM. Paris : Chez Louette, 1788 (vol. 2).

Dans les faits, toutes les cartes sont à prendre comme signe, mais toutes ne permettent pas un usage supplémentaire, cela même si elles le prétendent comme dans le cas de la carte fournie en fin du sixième volume in- $8^{\circ}$ du Guide pittoresque de chez Firmin Didot (fig. $\left.n^{\circ} 4\right)$. 


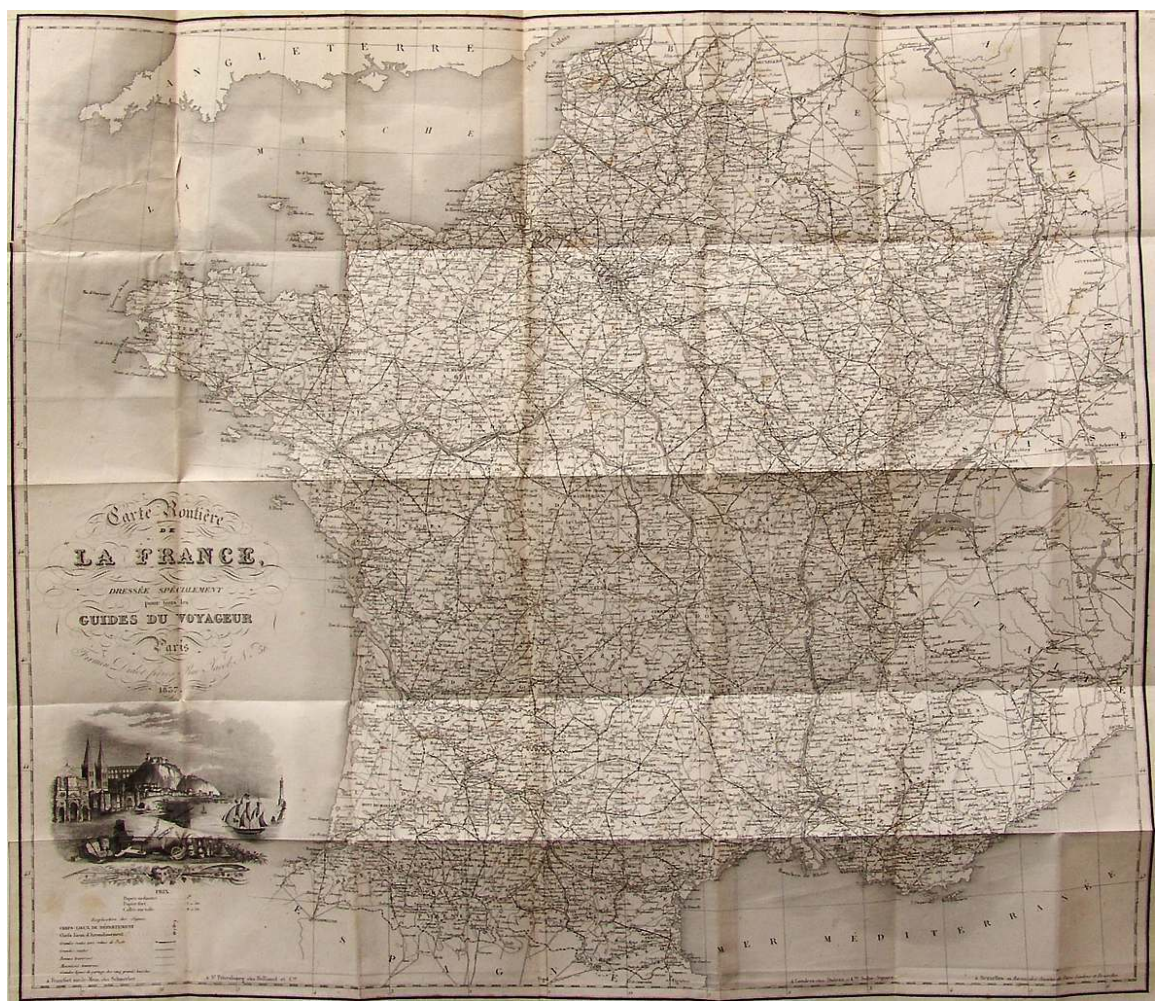

Carte routière de la France dressée spécialement pour tous les guides, 1837, in Guide pittoresque du Voyageur en France, contenant la statistique et la description complète des 86 départements, orné de 740 vignettes et portraits gravés sur acier, de 86 cartes de départements, et d'une grande carte routière de la France ; par une société de gens de lettres, de géographes et d'artistes. Paris : Firmin Didot frères, libraires, 1838, 6 vol. Angers, Bibliothèque. municipale, H 349.

Grande carte il est vrai puisqu'elle mesure $80 \mathrm{~cm}$ de côté, mais on imagine mal son maniement avec un autre volume que celui qui la contient, à l'intérieur d'une chaise de poste. On est plus en revanche du côté d'une possibilité de préparation du voyage. La «carte-signe " permet d'accéder à la posture du voyageur, et, au-delà d'élaborer un itinéraire. De ce point de vue la grande carte fournie à partir de 1774 dans les Livres de Poste offre un véritable outil pour tenter de confectionner son trajet. Dans ces trois derniers cas, on trouve une relation a minima entre cartes et textes dans le fait que la carte figure le contenu de l'ouvrage. Les routes des cartes de Louette, du Livre de Poste ou du Guide pittoresque... sont traitées dans le texte. Soixante ans plus tard, Adolphe Joanne dans son Itinéraire descriptif et historique de la Suisse préconise au touriste de " tracer [leur] itinéraire, tel est le premier devoir du voyageur. Pour qu'un voyage joigne l'utile à l'agréable, il faut qu'il ait été étudié... ${ }^{8}$ " Dès lors, le travail sur la carte apparaitt probable.

9 Le mot d'itinéraire, qui revient sans cesse dans ces guides, nécessite que l'on s'y intéresse un peu plus avant : en effet, son usage varie dans le temps. J'ai montré ailleurs qu'avant que le mot « réseau » ne s'impose pour exprimer ce concept, celui d'itinéraire avait été utilisé dans ce sens ${ }^{9}$. Par réseau, entendons une définition a minima : l'ensemble des lignes et des points dont l'articulation forme système. Or, les guides pris en tant qu'ensemble potentiellement homogène font montre quant à l'usage du concept d'une réelle indécision. Jusque vers les années 1780 , les choses semblent relativement simples. L'usage des «itinéraires » est rare et il semble concerner le niveau général d'organisation des 
routes. Ainsi, le Nouveau voyage de la France de Piganiol de la Force annonce, dès 1724 (et il ne changera pas par la suite), un itinéraire et des cartes, chaque carte renvoyant à une route. En cela, il ressemble fortement au Nouveau guide des chemins du royaume de France, contenant toutes ses Routes, tant générales que Particulières de Daudet publié en 1724, voire aux Livres de poste qui n'évoquent que des " routes " pour aller d'un point à un autre, ou encore à L'indicateur fidèle de Michel et Desnos des années 1760... Mais ces derniers ouvrages n'utilisent pas le mot « itinéraire ». En revanche, quelques ouvrages prenant la forme d'atlas comme celui d'Ogée en 1769, ou celui de Brion en 1766, voire celui de Le Rouge en $1759^{10}$ ont dans leur titre le mot "itinéraire " qui correspond à des cartes d'ensemble, et non à des cartes de trajets possibles. La carte de l'ensemble des voies et le concept d'itinéraire semblent donc pouvoir relever d'un même registre.

D'ailleurs, en dehors du cas de Piganiol de la Force, il semble que l'écrasante majorité des guides dont le titre comporte le mot « itinéraire » au moins jusqu'au début du XX $\mathrm{XX}^{\mathrm{e}}$ siècle, contiennent une carte générale. Pour un itinéraire de la France, la carte représente la France, pour l'itinéraire d'Orient, comme celui d'Isambert en 1881 dans la collection Guides Joanne, c'est une carte du bassin méditerranéen (fig. $\mathbf{n}^{\circ} 5$ ). Une définition complémentaire de ce qu'est un itinéraire apparait dans le guide d'Italie, paru chez Maison en 1855 : « Un itinéraire [...] n'est ni la description géographique complète d'un pays, ni un voyage où l'auteur peut [...] porter son attention sur tel côté pittoresque, moral, politique, industriel, artistique [...]. Un itinéraire doit donner à la fois moins et plus ; - plus car non seulement il doit fournir aux voyageurs une foule de renseignements pratiques, indispensables, mais il ne doit laisser dans chaque ville aucun objet remarquable ou curieux sans le signaler; - moins, car les longs développements lui sont interdits en général. » On est là dans la logique d'exposition d'un livre comme le Tableau de l'Égypte, de la Nubie et des lieux circonvoisins ou itinéraire à l'usage des voyageurs qui visitent ces contrées ${ }^{11}$. Finalement, Hélène Morlier propose une synthèse de cette approche dans laquelle l'itinéraire est un guide dans son ensemble débarrassé de ses scories ${ }^{12}$.

Figure 5

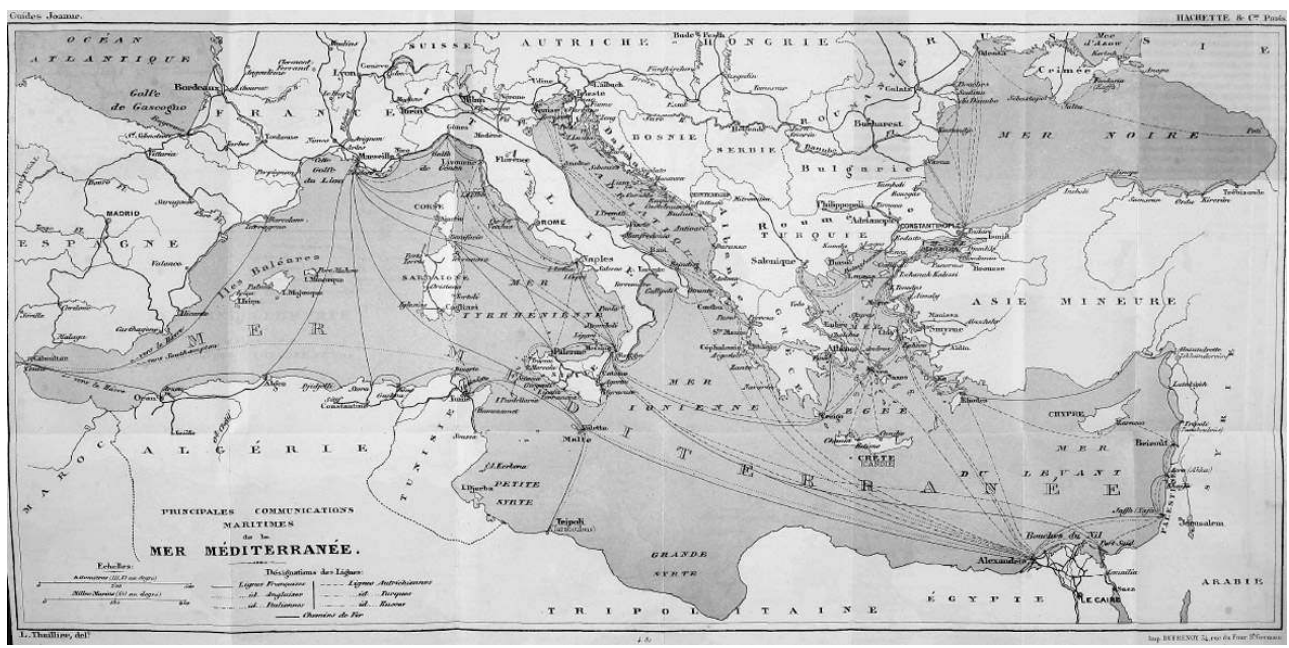

Principales communications maritimes de la mer Méditerranée, in ISAMBERT, Émile. Itinéraire descriptif, historique et archéologique de l'Orient. Paris : Librairie Hachette et Cie, 1881. Collection Guilcher.

11 La difficulté vient dans un deuxième temps, lorsque des ouvrages comme ceux de Joanne superposent deux niveaux d'itinéraires. Ainsi, l'Itinéraire de la Suisse... de 1857 articule cet 
itinéraire général avec des "modèles d'itinéraires» qui articulent des routes. Ces "modèles d'itinéraires" sont des voyages de plusieurs jours avec points de départ et d'arrivée. Il faut cependant attendre le milieu du $\mathrm{XX}^{\mathrm{e}}$ siècle pour voir dans le Guide Bleu de la France de 1956, au détour d'une page l'usage du mot itinéraire dans le seul sens que nous lui connaissons aujourd'hui: "L'itinéraire par Castres est beaucoup plus pittoresque ; l'itinéraire par Narbonne permet une plus grande vitesse et fait visiter trois des villes les plus importantes du Languedoc ${ }^{13}$. » Mais, à cette date l'itinéraire n'est plus une catégorie organisatrice du guide. Celui-ci s'est en partie trouvé remplacé par une autre catégorie : la route. Dans les années 1870, celle-ci se retrouve dans tous les grands guides européens quelle que soit la langue : ainsi, dans le Handbuch für Reisende, Belgien und Holland de Baedeker en 1873, les trajets sont numérotés de la route 1 à la route 51. Précisons ici qu'il s'agit d'une "route» qui peut aussi bien être une ligne de chemin de fer: la «route $n^{\circ} 5$ Von Lüttich nach Marloie» est signalée comme étant la «Ligne de l'Ourthe ». Dans le Handbook for travellers on the continent, part. 1 de Murray en 1875, on compte en tout 47 routes dans une vaste zone couvrant depuis la Hollande jusqu'à la Prusse. Les routes correspondent ici aussi bien au steamers qu'aux lignes de chemin de fer ${ }^{14}$. De même, dans le Guide Diamant sur la France de Joanne en 1873, on compte 565 routes possibles, principalement composées de trajets en chemin de fer.

Cette seconde échelle de préoccupation, qui va progressivement s'imposer dans un couple guide-route que la diffusion de l'automobile ne fera longtemps que renforcer correspond de son côté à une troisième production cartographique qui semble, d'après sa forme correspondre assez fortement à la pratique du déplacement. Alors que la norme cartographique impose l'orientation des cartes avec le nord en haut, bon nombre de ces cartes y dérogent, comme ces cartes militaires des XVII ${ }^{e}$ et XVIII ${ }^{e}$ qui placent l'objet de la conquête en haut de la carte ${ }^{15}$. Pour les voyages, l'exemple est d'abord à rechercher chez Piganiol de la Force tout au long du XVIII ${ }^{\mathrm{e}}$ siècle $^{16}$ (fig. $\left.\mathbf{n}^{\mathbf{0}} \mathbf{6}\right)$. 
Figure 6

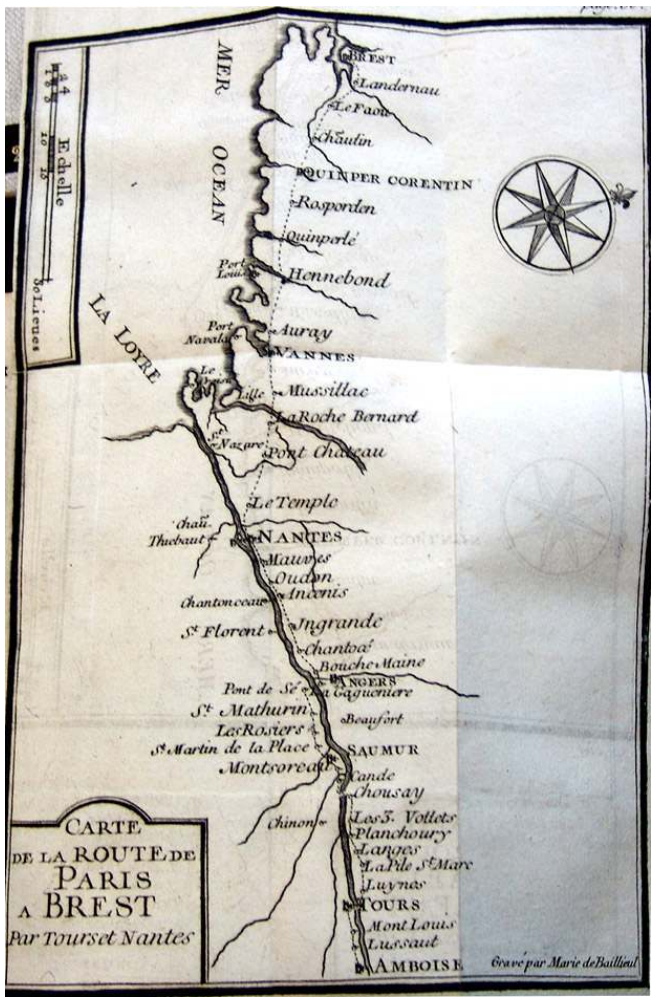

PIGANIOL DE LA FORCE. Nouveau voyage de la France avec un itinéraire et des cartes. Paris Théodore Legras, 1724, p. 72.

13 Mais ce type de cartographie se maintient: tant dans des guides relatifs à un trajet unique, comme c'est le cas du Guide du voyageur sur le canal du midi de Georges de Riquet en 1836 (fig. $\mathbf{n}^{\circ} 7$ ), sorte de guide de tourisme industriel avant la lettre ${ }^{17}$, que dans un guide de Paris au Havre en chemin de fer, comme c'est le cas du Guide pittoresque de 1853 (fig. $\left.\mathbf{n}^{\circ} \mathbf{8}\right)$...

Figure 7

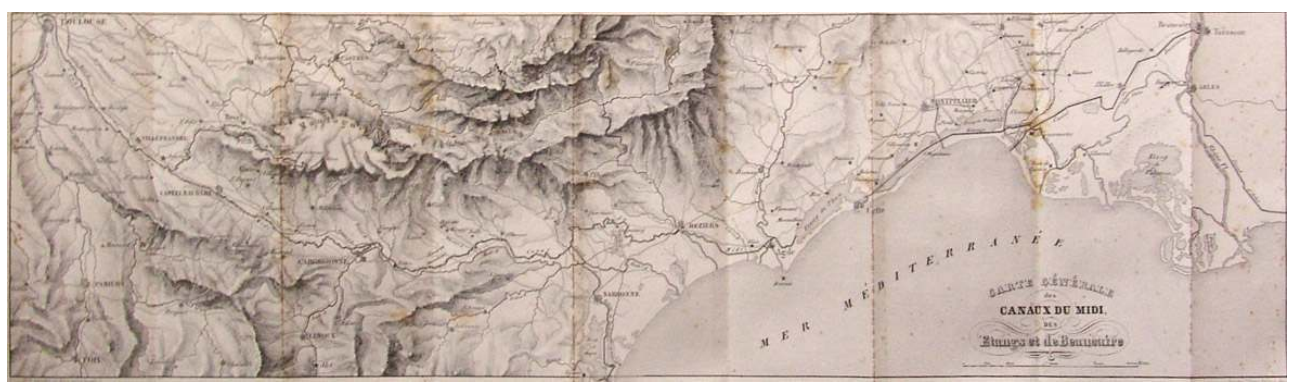

CARAMAN, RIQUET Georges (Cte de). Guide du voyageur sur le canal du midi et ses embranchements et sur les canaux des étangs et de Beaucaire; par M. le Comte G. de C ${ }^{\star \star \star}$. Toulouse : Imprimerie de Jean Matthieu Douladoure, rue Saint Rome, n 41, 1836. Angers, Bibliothèque. municipale, 103870. 
Figure 8

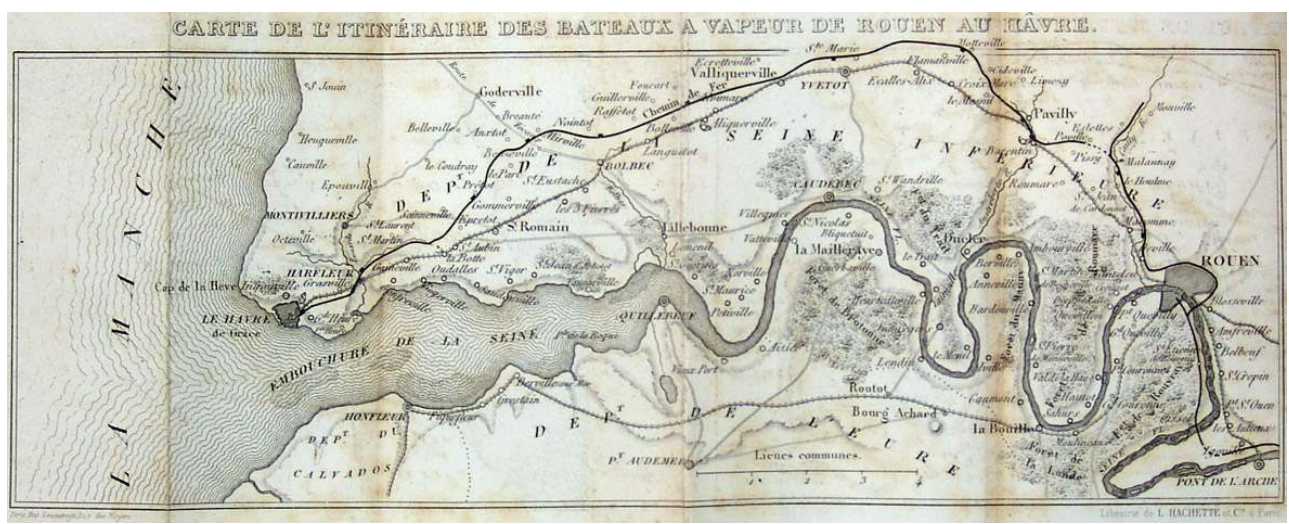

Petit itinéraire du Chemin de Fer de Paris au Havre. Paris : Hachette, s.d. (carte n³). Dans le développement il est indiqué : Guide pittoresque, Hachette, 1853. Coll. part.

14 Mais on le trouve également dans des guides généraux qui dans ce cas, à l'exemple de ce qu'avait fait Piganiol de la Force (fig. $\mathbf{n}^{\circ} \mathbf{9}$ ), font se succéder des cartes de trajet suivies de leurs explications.

\section{Figure 9}

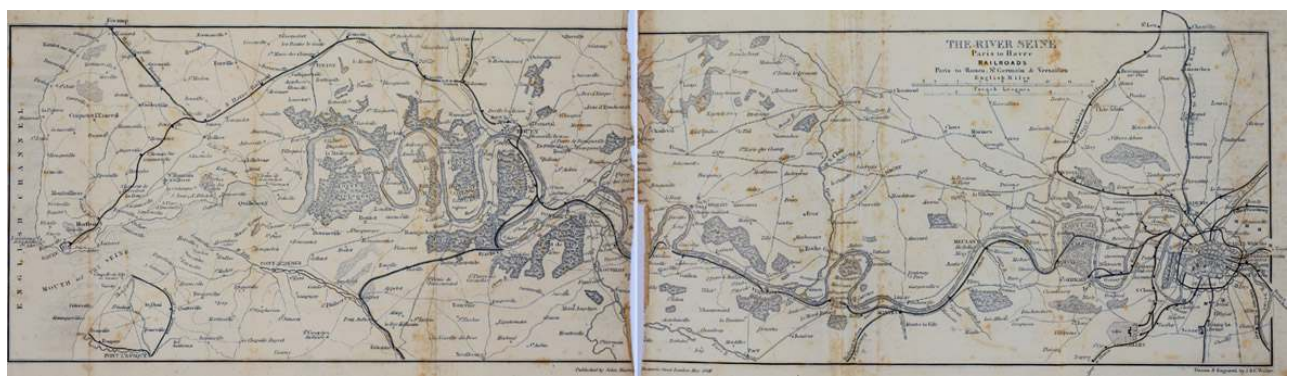

MURRAY, John. A hand-book for travellers in France: being a guide to Normandy, Brittany; the Rivers Seine, Loire, Rhône, and Garonne ; the French Alps, Dauphiné, The Pyrenees, Provence and Nice, etc ; The Railways and Principal Roads, with maps and Plans of towns. London : John Murray, 1867. Collection Guilcher.

On le retrouve encore au début du XXe siècle dans le Guide Joanne Dieppe, Le Tréport, Mers, Ault, Onival... de 1907 (fig. nº10). 


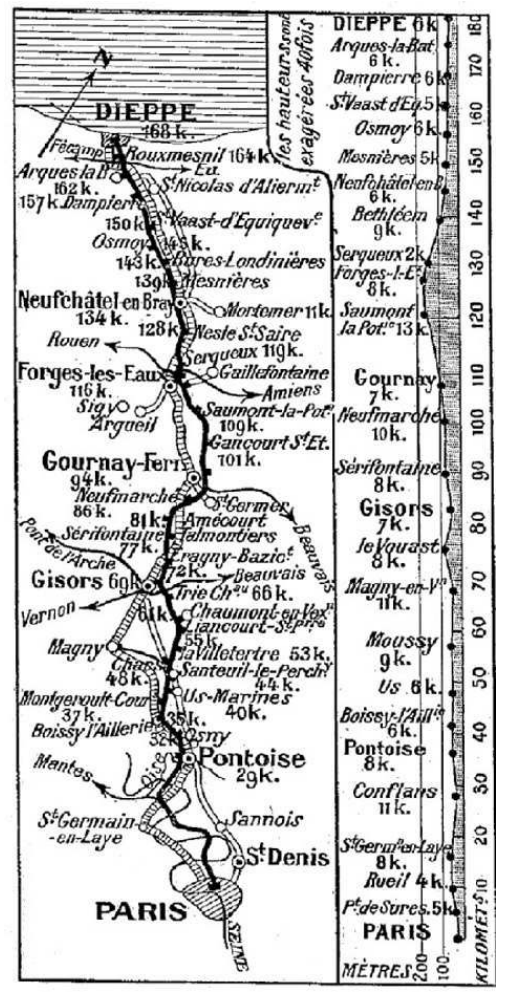

Guide Joanne. Dieppe, Le Tréport, Mers, Ault, Onival. Paris : Hachette, 1907, p. 14

Si ce type de carte disparaît des Guides Bleus des années 1950, on le retrouve dans les éditions plus récentes : même si l'orientation (nord en haut) est maintenant respectée, le Guide Bleu sur la France de 1997 utilise ce type de représentation (fig. nº11).

Figure 11

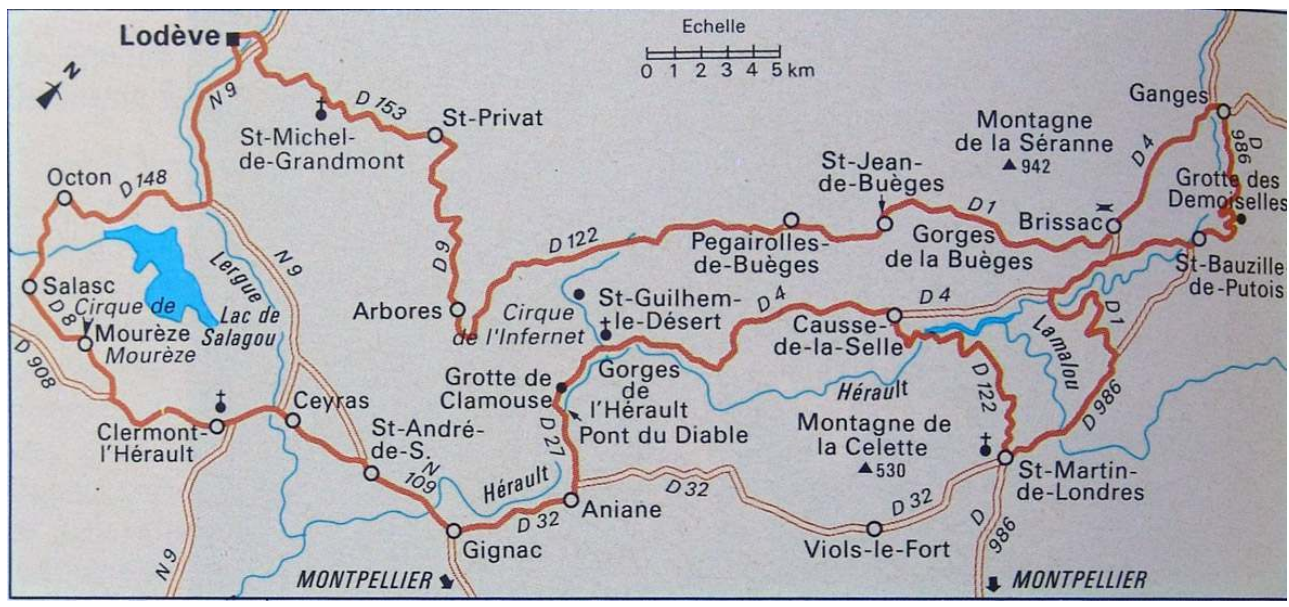

Guide Bleu, France. Paris : Hachette, 1997, p. 416. @ Guide Bleu/France/, Hachette, 1997.

C'est dire si cette forme en bande est considérée comme efficace. À l'origine, ce type de représentation renvoie à des formes cartographiques anciennes liées au déplacement, comme les relations de pèlerinages médiévaux ${ }^{18}$. Mais celles-ci semblent avoir été actualisées dans les travaux relatifs aux routes, qu'il s'agisse de cartes à usage restreint, 
comme pour l'Atlas Trudaine pour les Ponts et Chaussées, ou pour les guides, comme dans le Nouveau voyage de la France de Piganiol de la Force, ou un peu plus tard, L'indicateur fidèle de Michel et Desnos qui paraît à partir de 1762 ; voire le Conducteur français de Denis, publié en 1776.

L'une des particularités de ces cartes en bandes est qu'elles sont produites en fonction d'un usage prédéterminé. Le lien entre usage et production est tel que dans certains cas, comme ceux de L'indicateur fidèle ou du Conducteur français, les cartes sont censées se suffire à elles-mêmes. Comme l'annonce le prospectus de L'indicateur fidèle :

«Les cartes de ces routes se vendent ensemble ou séparément par feuilles détachées, et leur forme est portative. Le voyageur peut aisément en enfermer une dans un portefeuille, et la consulter au besoin. Elles éclairent, elles dirigent, et elles mettent celui qui en est possesseur, dans le cas de se passer de tous les renseignements que l'on recherche dans les voyages; enfin, elles sont entièrement consacrées à l'utilité publique, et au besoin de tous ceux qui voyagent. » En effet : «Communément, celui qui entreprend une route consulte ceux qui l'on faite avant lui, s'il est en chemin il s'informe, à mesure qu'il avance, du nombre des lieues qui lui restent à faire, des endroits où il peut prendre ses repas, ou passer le temps de la nuit, et rarement on lui donne là-dessus des réponses précises. Avec une de nos Cartes, le Voyageur n'a aucun besoin de toutes ces demandes; il voit tous les endroits par lesquels il doit passer, et une juste mesure de leur éloignement respectif ; il connaît en même temps les Bourgs, les Villages, les Hameaux, les Fermes, les maisons Religieuses, les Bois, les Prés, les Avenues, les Rivières, les Ponts, les Gués, les Ruisseaux, les Étangs, et les Marais, enfin jusqu'aux Montagnes et aux Plaines qu'il a à traverser ${ }^{19}$.»

On trouve ici la même attention que celle que l'on trouvera 90 ans plus tard chez A.J. du Pays dans son Guide d'Italie de 1855 qui souhaite donner une information utile et synthétique. Les moments de relation intime entre cartes et textes qui cassent le modèle orienté au nord, tout en réduisant l'espace à une bande - peut-être paysagère - canalisent le lecteur dans une forme de déplacement sans autre alternative que celles que le guide accepte de lui concéder. Sorti de la figuration on est dans le blanc, terra incognita des cartographes laissée aux hardis explorateurs.

21 Quoi qu'il en soit, cette cartographie, qui ne reprend pas les cartes de France déjà publiées, a un coût tel que par exemple les Guides Joanne, après avoir un temps utilisé les fonds des guides publiés par Louis Maison ${ }^{20}$, en arrivent à des cartes par zones, plus faciles à utiliser dans différents guides et qui permettent par ailleurs la publication d'un Atlas historique et statistique des chemins de fer français ${ }^{21}$. On notera que ce faisant, la relation entre texte et carte se distend. En effet, la figuration de la carte outrepasse amplement la liste des éléments décrits dans le texte. Il faut ensuite attendre de nouveaux usagers comme les cyclistes et automobilistes pour qu'une cartographie innovante voit le jour en tentant de coller à leurs besoins. Celle-ci, en juxtaposant les profils du relief routier avec les trajets proposés, permet de connaître les difficultés pratiques, entendons la pente qui force l'excursionniste à descendre de vélo, lors de la promenade ${ }^{22}$. La mise en page dit ici beaucoup, tant le texte se trouve compressé sur le côté de la carte. La relation a donc été construite bien au-delà du simple ajout d'une carte en fin d'ouvrage ou entre deux cahiers. Ici, la carte ne peut aisément se vendre séparément du livre. C'est probablement au sein de ces usages très spécifiques que le mot itinéraire, en englobant un seul trajet, passe de l'échelle du général à celle du particulier. 
Si l'on s'intéresse maintenant aux relations explicites qu'entretiennent cartes et textes dans ces guides, les résultats sont pour le moins insatisfaisants. Piganiol de la Force ne renvoie pas dans son texte aux cartes qu'il intègre. Il évoque bien dans son introduction l'innovation qui consiste à avoir « ajouter des itinéraires et des cartes faites exprès, afin que les Voyageurs eussent dans un seul Volume de quoi s'amuser et s'instruire» (p. V.) Mais ensuite, malgré le fait que les cartes soient mises en tête de chapitre à raison d'un chapitre par route, il n'en est plus fait mention. Inversement, L'indicateur fidèle de Michel et Desnos produit des cartes sans texte. Chez Louette, qui publie son Itinéraire complet de la France en 1788, le titre précise très justement que l'ouvrage est Orné d'une Carte Géographique, car la seule mention de cette carte dans le texte se trouve dans les instructions préliminaires pour préciser que la lieue utilisée mesure « 2000 toises, dans tout l'ouvrage, telle que l'annonce l'échelle de notre Carte.» (p. VI). Le texte des Livres de Poste n'évoque pas la carte, cela même dans des éditions tardives des années 1830. Le Petit itinéraire du chemin de fer de 1853 ne fait pas mieux. Étrangement, le Guide Diamant renvoie à de nombreuses cartes d'état-major, mais pas à celles qu'il fournit. Joanne, s'il renvoie à la carte, ne souhaite pas «forcer le voyageur à avoir sans cesse une carte à la main $^{23}$.» Enfin, le Guide Bleu de la France de 1956, très pauvre en cartes il est vrai n'y renvoie pas non plus. Le seul ouvrage à évoquer les cartes, et encore rarement, est le Murray. Dès la version du guide sur l'Europe de 1838, l'introduction précise : «Each route is numbered with Roman numerals, corresponding with the figures attached to the Route on the Map, which thus serves as an Index to the Book; at the same time that it presents a tolerably exact view of the great high roads of Europe, and the course of public conveyances. » L'édition de 1853, lorsqu'elle évoque la question des nouvelles lignes de chemin de fer en Allemagne, précise : "Railroads have extended rapidly in Germany within the last few years. The map, and the following routes, will show what lines are now open, or about to open ${ }^{24}$. " On notera de ce point de vue, que cette série de guides est celle qui semble continuer à utiliser des cartes en bandes le plus tardivement, alors que les autres séries ont abandonné pour un temps cette pratique. Comme si l'effort de production cartographique à destination des guides amenait à tenter de mettre mieux en évidence la relation entre cartes et textes.

Mais existe-t-il une relation moins visible, qui assurerait la relation du texte et de la carte ? La difficulté réside ici dans le fait que le processus ne lie pas seulement usage de la cartographie et forme des guides. Un autre élément, nécessairement présent, est la captivité relative au réseau matériel des voies. Un guide qui ne s'appuie que sur la malleposte ou le chemin de fer, surtout lorsque ces réseaux sont peu denses, produit presque nécessairement un discours sur chaque route, voire sur chaque ligne. Trois respirations au moins sont aisées à repérer : au début du XVIII siècle pour la «malle-poste » de Piganiol de la Force; dans les années 1850 pour le chemin de fer dans différents guides, voire peut-être les bonnes routes pour les premières automobiles au début du $\mathrm{XX}^{\mathrm{e}}$ siècle. La densification d'une part, et la diffusion de l'usage du concept de réseau, en partie du fait de la carte, complexifie les choses. On peut alors avoir des descriptions par sousréseaux, comme chez Joanne dans les années 1860. En même temps la multiplication des lignes mène à un calcul comptable qui fait de la multiplication des cartes bandes un gouffre financier. Le fait que le projet du Conducteur Français de Louis Denis, commencé en 1776 n'ait jamais été achevé (seules 52 cartes ont été fournies) - alors que le réseau en arrivait à un niveau de densité très élevé ${ }^{25}$ - est un indice de la fragilité d'une telle aventure éditoriale. La carte générale, gravée à partir d'autres, est un investissement nettement moins onéreux. Posons alors la question inverse : l'usage d'une carte générale, 
à l'échelle de la France par exemple, a-t-elle des conséquences sur la structure des ouvrages? Pour esquisser une réponse je m'appuierai ici sur une remarque d'Hélène Morlier à propos de la mutation de la structure des Guides Joanne dans les années 1860 . Décrivant la mise en place de la nouvelle série intitulée "Itinéraire Général de la France ", elle écrit: "Il s'agit de passer d'un système descriptif linéaire à un autre traitant de surfaces. Le projet prend du temps à se mettre en place, mais dès 1865 , c'est chose faite...26. » Á ses yeux, l'évolution est en partie liée à l'affranchissement de la tutelle de la Bibliothèque des Chemins de fer, ce qui semble une hypothèse solide. Cependant c'est aussi à ce moment que les cartes fournies dans les guides passent de la bande à la carte régionale. Sans prétendre démêler qui de la poule ou de l'œuf était avant, on a là un aspect supplémentaire du changement de l'espace des guides. De ce point de vue, le retour à l'ordre alphabétique est un indice fort en faveur d'une structuration différente de cet espace.

Quels espaces des guides ressortent de cette rapide enquête? La question est rendue d'autant plus complexe que, comme nous l'avons vu, les figurations de l'espace que sont les cartes, si elles sont présentes, n'en sont pas pour autant nécessairement reliées de façon explicite aux figurations textuelles contenues dans les guides. On peut cependant tenter d'en présenter quelques types. Le premier est le contenant que représente l'ouvrage avec sa représentation iconique d'un pays ou d'une contrée quelle qu'elle soit. La morphologie même du livre, qui bien souvent place la carte en début ou en fin d'ouvrage, voire sur la troisième de couverture, montre cet aspect englobant en même temps que délimitant. Le deuxième est celui d'un voyage structuré en forme de lignes passant par une collection de points. En d'autres mots, l'itinéraire tel que nous l'entendons aujourd'hui ; cela même si on y ajoute l'ensemble des éléments visibles depuis ce filaire. La difficulté à dire la mobilité se trouve ici remplacée par le principe de la succession de points de passages. Le temps du voyage se trouvant remplacé par l'espace de la carte. Les guides suivant un classement alphabétique des lieux restreignent fortement cet aspect. À ce propos, Adolphe Joanne dans son Itinéraire de la Suisse... écrivait que cet ordre "nécessite des recherches continuelles et force le voyageur à avoir sans cesse une carte à la main. » Un Guide Bleu relativement récent comme celui publié en 1997, suit l'ordre alphabétique par région, mais s'appuie sur une collection de plus de 300 cartes pour palier la difficulté. Le troisième type d'espace est celui du réseau dans son ensemble qui articule plusieurs itinéraires. La plupart des guides de la France montrent de ce point de vue une conception constante qui fait partir de Paris la plupart des routes, montrant en cela une prise en compte tout à fait consciente de l'aspect étoilé du système français. Le quatrième type d'espace, dont il a à peine été question ici est régional. Il peut être celui des sous-réseaux, avant la mise en place de la SNCF, ou constitutif de régions parfois héritées de ces découpages territoriaux. Le plus bel exemple s'en trouve certainement dans la seconde carte présente dans le Guide Bleu de la France de 1956 qui organise son discours et la collection des guides sur ce modèle, passant ainsi d'une logique linéaire à une logique zonale (fig. $\left.\mathbf{n}^{\circ} \mathbf{1 2}\right)$. 


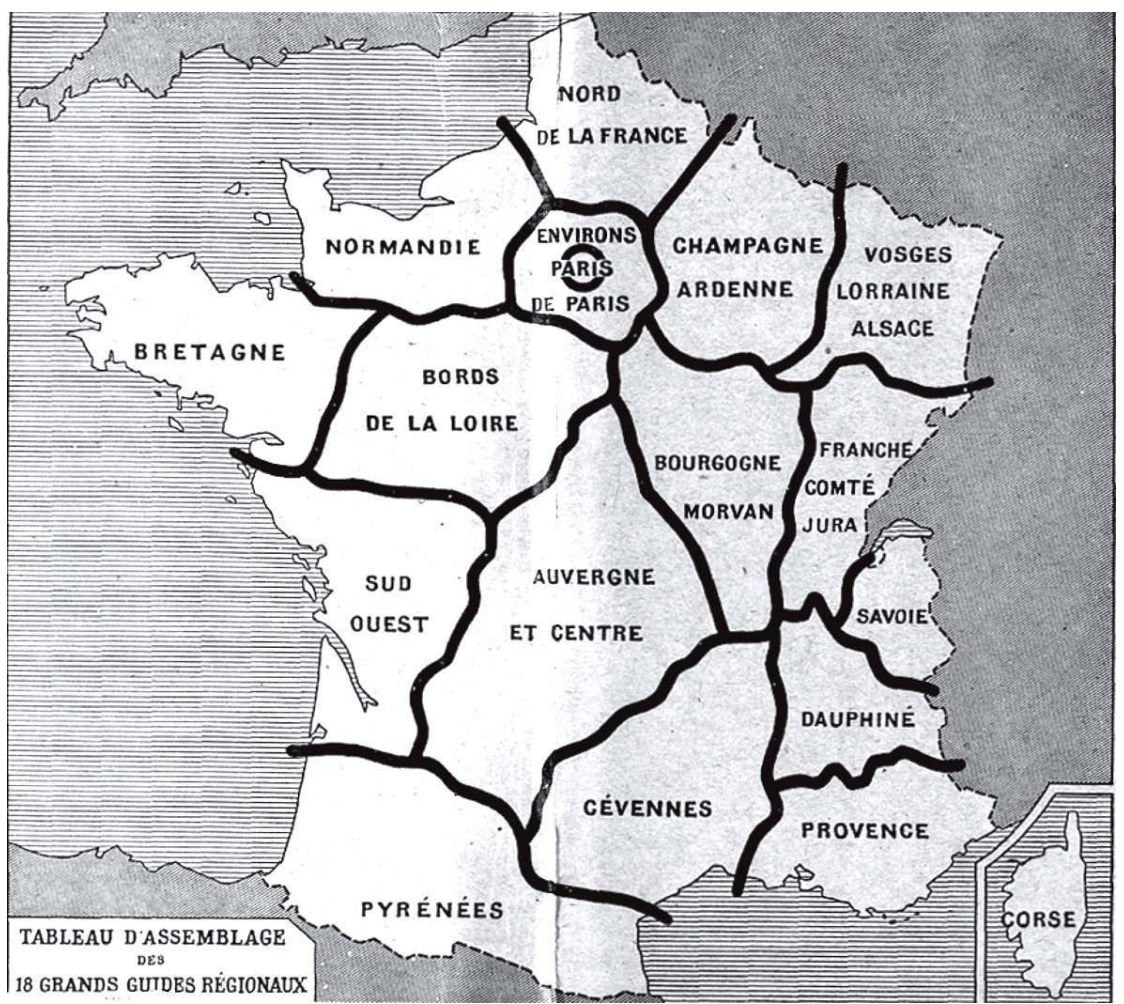

Deuxième de couverture du Guide Bleu pour la France. France en un volume, Les Guides Bleus. Paris Hachette, 1956.

(c) Guide Bleu/France/, Hachette, 1956.

Enfin, le cinquième type, peu présent dans notre échantillon, est le système ponctuel, on ne le trouve à ma connaissance que dans la collection des guides bleus récents qui offrent, en début de volume une série de cartes donnant à voir les lieux de « la France Romane ", des "plus beaux châteaux », de "l'architecture moderne » ou des « grands musées ». En cela, ces guides dépassent la simple liste, alphabétique ou pas, pour affirmer que la mise en série des lieux fabrique l'espace de la contrée qu'ils dépeignent.

Les guides sont donc des objets complexes : ils articulent des textes de différents types, des tableaux, des index, des gravures, voire des photos et des cartes. L'ensemble ainsi formé apparaît très hétérogène. Ce serait cependant une erreur que d'en rester à cette première impression. Les choix, même lorsqu'ils apparaissent comme étant le fruit de micro-décisions - je pense par exemple ici à ce guide des chemins de fer de 1853 qui lie trois cartes de différentes factures, et trois textes d'auteurs différents, dans le cadre d'un seul ouvrage - se rattachent bien souvent à des logiques plus amples. La présence d'une « carte géographique » dans un itinéraire apparaît quasi-obligatoire à cette époque. Cela permet de s'intéresser à l'utilité de la carte telle qu'elle peut avoir été perçue par les auteurs. Soit, celle-ci peut permettre de se repérer, mais bien souvent ses fonctions sont autres: donner une légitimité qui renvoie à des savoirs savants, se raccrocher à un corpus, comme celui des textes géographiques, donner à voir la portée d'un ouvrage... et permettre l'organisation d'un voyage à partir de ce que le monde savant appellerait un graphe. Cela permet également de revenir sur les conséquences de ces usages. Alors que les géographies de la France, souvent coûteuses, connaissent une diffusion restreinte, les 
guides meilleurs marchés offrent, avant - puis avec l'almanach des Postes - une figuration de la France sous la forme d'une carte. Il faudra ensuite attendre l'enseignement scolaire obligatoire et sa géographie pour propager cette image sur le temps long. Au-delà, c'est l'usage du réseau que la diffusion de ces cartes thématiques car elles se concentrent sur les voies de communication - rend possible.

\section{NOTES}

1. - Nous tenons à remercier le Musée de la Poste pour l'autorisation de reproduction des figures 2, 3, 6; la Bibliothèque Municipale d'Angers pour les figures 4 et 7, et Goulven Guilcher pour les figures 5 et 9 .

2. - Sur ce point, on lira: BONIN, Serge. La France de Charles Estienne. Annales. Économies, Sociétés, Civilisations, année 1961, vol. 16, nº 6, p. 1121-1130.

3. - GRITTI, Jules. Les contenus cultures du Guide bleu. Communications, 1967, vol. 10, $\mathrm{n}^{\circ} 1$, p. 51-64. Le texte est en partie en réaction au texte virulent de Roland Barthes sur le Guide Bleu : BARTHES, Roland. Le «Guide bleu ». Mythologies. Paris : Seuil, 1957 (p. 113-117 de l'édition de 1970). On verra également: SAUNIER, Pierre-Yves. Le guide touristique, un outil pour une possible histoire de l'espace: Autour des guides de Lyon, 1800-1914. Géographie et cultures, $1995, \mathrm{n}^{\circ} 13$, p. 35-54.

4. - Sur ce point : BELLEFON (de), Renaud. Des autochtones pris dans les filets des guides-livres : les guides de montagne XIX ${ }^{\mathrm{e}}-\mathrm{XX}^{\mathrm{e}}$ siècle, in CHABAUD, Gilles, COHEN, Évelyne, COQUERY, Natacha, PENEZ, Jérôme (dir.). Les guides imprimés du $X_{V I}{ }^{e}$ au $X X^{e}$ siècle, Villes, paysages, voyages. Paris : Belin, 2000, p. 259-270.

5. - Sur ce point, et à propos des guides Joanne, Hélène Morlier parle de stocks de cartes utilisés tant qu'ils ne sont pas épuisés. MORLIER, Hélène. Les Guides-Joanne, genèse des Guides-Bleus. Itinéraire bibliographique, historique et descriptif de la collection de guides de voyage (1840-1920). Paris : Les sentiers débattus, 2007, p. 57.

6. - Sur ce point on consultera les notices 165 à 169 et 180 de MORLIER, Hélène. Les GuidesJoanne, genèse des Guides-Bleus. Itinéraire bibliographique, historique et descriptif de la collection de guides de voyage (1840-1920). Paris : Les sentiers débattus, 2007.

7. - MARIN, Louis. Les voies de la carte, in Cartes et figures de la terre. Paris : Centre Georges Pompidou, 1980, p. 47-54.

8. - JOANNE, Adolphe. Itinéraire descriptif et historique de la Suisse et du Jura français de Baden-Baden et de la Forêt noire ; de la Chartreuse de Grenoble et des eaux d'Aix ; du Mont Blanc, de la vallée de Chamouni, du Grand Saint-Bernard et du Mont-Rose ; avec une carte routière imprimée sur toile. Paris : Paulin, 1841.

9. - VERDIER, Nicolas. Le réseau technique est-il un impensé du XVIII ${ }^{\mathrm{e}}$ siècle : le cas de la poste aux chevaux. Flux, Cahiers scientifiques internationaux Réseaux et Territoires, 2007, $\mathrm{n}^{\circ} 68$, p. 7-21.

10. - OGÉE. Atlas itinéraire de Bretagne contenant les cartes particulières de tous les grands chemins de cette province avec tous les objets remarquables qui se rencontrent à une demie lieue adroite et agauche. Nantes: Chez l'auteur, 1769 ; BRION DE LA TOUR. Atlas itinéraire de l'Europe, adapté, quant à la France aux messageries royales, nouvelles diligences..., par le Sieur Brion. Paris : Brion, 1766 ; LE ROUGE (trad.). Itinéraire de toutes les 
routes de l'Angleterre. Paris : Desnos, 1759, réédité en 1766. Puis vient la série des Indicateurs fidèles... de DESNOS, publiés et republiés de 1764 à 1775.

11. - RIFAUD, J.J. Tableau de l'Égypte de la Nubie et des lieux circonvoisins ; ou itinéraire à l'usage des voyageurs qui visitent ces contrées. Paris : Treutel et Würtz libraires, 1830.

12. - DU PAYS, A.J. Itinéraire descriptif, historique et artistique de l'Italie, enrichi de 22 cartes et plans. Paris : Maison, 1855 (le texte est cité par MORLIER, Hélène. Les Guides-Joanne, genèse des Guides-Bleus. Itinéraire bibliographique, historique et descriptif de la collection de guides de voyage (1840-1920). Paris : Les sentiers débattus, 2007, p. 39-40).

13. - AMBRIERE, Francis (dir.). France en un volume. Les Guides Bleus. Paris: Librairie Hachette, 1956, p. 791.

14. - La traduction du mot route en français renvoie immanquablement à direction ou itinéraire... 15. - Sur ce point, on lira SAHLINS, Peter. Frontières et identités nationales, la France et l'Espagne dans les Pyrénées depuis le XVII' siècle. Paris : Belin, 1996, p. 51-60.

16. - La même carte se trouve dans la dernière édition de 1780.

17. - CARAMAN, RIQUET Georges (Cte de). Guide du voyageur sur le canal du midi et ses embranchements et sur les canaux des étangs et de Beaucaire; par M. le Comte G. de $\mathrm{C}^{* * *}$. Toulouse : Imprimerie de Jean Matthieu Douladoure, rue Saint Rome, $n^{\circ} 41,1836$.

18. - GAUTIER-DALCHÉ, P. De la liste à la carte : limite et frontière dans la géographie et la cartographie de l'Occident médiéval. Castrum 4, Frontière et peuplement dans le monde méditerranéen au Moyen Âge. Rome: École française de Rome/Casa de Velasquez, 1992, p. 19-29.

19. - Prospectus du guide des voyageurs pour les routes royales \& particulières de la France \& autres, in L'indicateur fidèle ou guide des voyageurs dressé par les sieurs Michel et Desnos, ingénieurs géographes du Roy. $3^{\text {ème }}$ édition corrigée et considérablement augmentée en 1767 et nouvellement parue.

20. - MORLIER, Hélène. Les Guides-Joanne, genèse des Guides-Bleus. Itinéraire bibliographique, historique et descriptif de la collection de guides de voyage (1840-1920). Paris : Les sentiers débattus, 2007, p. 57-75.

21. - JOANNE, Adolphe. Atlas historique et statistique des chemins de fer français, contenant 8 cartes gravées sur acier. Paris : Librairie de L. Hachette et Cie, 1859.

22. - On notera de ce point de vue qu'au même moment les cartes routières Taride inventent des figurés de pente des routes sur leurs cartes...

23. - JOANNE, Adolphe. Itinéraire descriptif et historique de la Suisse et du Jura français de Baden-Baden et de la Forêt noire ; de la Chartreuse de Grenoble et des eaux d'Aix ; du Mont Blanc, de la vallée de Chamouni, du Grand Saint-Bernard et du Mont-Rose ; avec une carte routière imprimée sur toile. Paris : Paulin, 1841, p. XII. Ce texte est cité par NORDMAN, Daniel. Les guides Joanne, in NORA, Pierre (dir.). Les Lieux de mémoire. La Nation. Paris : Gallimard, 1986, t. 2, vol. 1, p. 529-558.

24. - MURRAY, John. A hand-book for travellers on the Continent: being a guide through Holland, Belgium, Prussia and Northern Germany, and along the Rhine, from Holland to Switzerland... with an index map. London : John Murray and Son, 1838, p. XLVII et 1853, p. 207. 25. - BRETAGNOLLE, Anne, VERDIER, Nicolas. L'extension du réseau des routes de poste en France, de 1708 à 1833, in LEROUX, M. (éd.). Postes d'Europe, XVIII ${ }^{\mathrm{e}}-\mathrm{XXI}^{\mathrm{e}}$ siècle, jalons d'histoire comparée - histoire des réseaux postaux en Europe du XVIII ${ }^{\mathrm{e}}$ au $\mathrm{XXI}^{\mathrm{e}}$ siècle Paris : Comité pour l'histoire de la Poste, 2007, p. 155-172.

26. - MORLIER, Hélène. Les Guides-Joanne, genèse des Guides-Bleus. Itinéraire bibliographique, historique et descriptif de la collection de guides de voyage (1840-1920). Paris : Les sentiers débattus, 2007, p. 170. 


\section{RÉSUMÉS}

La relation entre cartes et guides est ancienne en France. Cet article interroge cette relation en s'intéressant d'abord à la nature des cartes contenues dans les guides, puis essaie de mettre en avant la relation existant entre forme de la carte et forme du texte dans le guide. C'est principalement au travers de la relation aux cartes en bandes que la question est ici développée. Le corpus étudié commence au début du XVIII ${ }^{\mathrm{e}}$ siècle pour s'achever à la fin du XX ${ }^{\mathrm{e}}$ siècle.

The relations between maps and guides are old in France. This article questions this relation by being first interested in the nature of maps contained in the guides, then try to put forward the relation existing in the form of the map and the form of the text in the guide. It is mainly through the relation with the maps in bands that the question is developed here. The studied corpus starts at the beginning of the XVIIIth century to end at the end of the XXth century.

\section{INDEX}

Mots-clés : géographie, guide, Guide Bleu, Guide Joanne, Guide Murray, histoire de la cartographie, itinéraire, Louette, Piganiol de la Force, réseau, route

\section{AUTEUR}

\section{NICOLAS VERDIER}

chargé de recherche au CNRS, UMR 8504 Géographie-cités, nicolas.verdier@parisgeo.cnrs.fr 